\title{
Observation of photon noise by cold-electron bolometers
}

\author{
A. V. Gordeeva, ${ }^{1,2,3, a)}$ V. O. Zbrozhek, ${ }^{1}$ A. L. Pankratov, ${ }^{1,2,3}$ L. S. Revin, ${ }^{1,2,3}$ \\ V. A. Shamporov, ${ }^{1,2,3}$ A. A. Gunbina, ${ }^{1}$ and L. S. Kuzmin ${ }^{1,4}$ \\ ${ }^{1}$ Nizhny Novgorod State Technical University n.a. R.E. Alekseev, GSP-41, Nizhny Novgorod 603950, Russia \\ ${ }^{2}$ Institute for Physics of Microstructures of RAS, GSP-105, Nizhny Novgorod 603950, Russia \\ ${ }^{3}$ Lobachevsky State University of Nizhny Novgorod, GSP-20, Nizhny Novgorod 603950, Russia \\ ${ }^{4}$ Chalmers University of Technology, 41296 Gothenburg, Sweden
}

(Received 19 October 2016; accepted 3 April 2017; published online 20 April 2017)

\begin{abstract}
We have observed the photon noise by measuring a response to the black body $350 \mathrm{GHz}$ radiation and noise of the cold-electron bolometers (CEBs). The experimental results have been fit to the theoretical model of CEBs with two heat-balance equations. The measured noise has been decomposed into several terms with the help of theory. It is demonstrated that the photon noise exceeds any other noise components, which allows us to conclude that the bolometers measure the photon noise. Moreover, a peculiar shape of the noise dependence on the absorbed power originates completely from the photonic component according to the theory. In the additional experiment on heating of the cryostat plate together with the sample holder, we have observed the near independence of the noise on the electron temperature of the absorber, which has provided another proof of the presence of the photon noise in the first experiment. The least ratio between internal and photon noise equivalent powers, observed in our experiments, is 1.1 for the absorbed power of $1-2 \mathrm{pW}$. Published by AIP Publishing. [http://dx.doi.org/10.1063/1.4982031]
\end{abstract}

The current cosmological experiments apply very strict requirements to the detectors installed on telescopes. The Cosmic Microwave Background (CMB) is still the target of many Cosmology experiments. The main goal of the current cosmology is the understanding of the inflationary process that occurred when the universe was about $10^{-37} \mathrm{~s}$ old, according to the Standard Model. Many theoretical models predict that this process should have left footprints of it in the form of a very small polarized signal called B-modes. Detecting this signal would provide important information about the primordial universe and the high energy physics.

The recent joint analysis of BICEP2 experiment and Planck satellite results confirms that BICEP2 did not detect any B-modes of cosmological origin. ${ }^{1}$ This work has demonstrated the stringent necessity of developing sensitive multifrequency $\mathrm{CMB}$ experiments to correct the observations from the dust contributions. Cold-electron bolometers $(\mathrm{CEBs})^{2,3}$ are promising detectors for cosmological applications, as they have all the qualities necessary to perform tasks such as the high sensitivity to terahertz radiation and immunity to cosmic rays. ${ }^{4}$

The purpose of this work is experimental demonstration that cold-electron bolometers have an ultimate sensitivity, i.e., sensitive to the photon noise. Photon noise contains a contribution due to a shot noise mechanism and is inevitably present in any radiation due to the discrete nature of the photons. The photon noise turns into a voltage noise of the detector, multiplied by the volt-watt response $S_{V}$ of the receiver. Ideally, all the other components of the detector noise, including an internal noise, must be smaller than the photonic component. If this condition is met, the detector is limited by the photon noise. Experimental demonstration of the photon noise is necessary for the installation of this type



of bolometers on the next generation of balloon and spacebased telescopes.

In this paper, we present the results of optical experiments with parallel-series arrays of cold-electron bolometers (CEBs) ${ }^{5,6}$ In order to obtain the main characteristic of detectors - the noise equivalent power (NEP), one needs to know the absorbed power. At least three different approaches are used to determine the absorbed power in cold electron bolometers:

1. from electromagnetic properties of the device, if the efficiency of antenna absorption is known. This approach was used, for example, in Ref. 6;

2. from simplified heat-balance equation at zero bias, where only two terms are nonzero. It was used, for example, in Refs. 7 and 8;

3. from the full set of heat-balance equations and fitting of the whole IV-curves. ${ }^{9}$

We have shown the limitations of the first method in Ref. 9. The second method can give a misleading result due to the ambiguity of the zero-bias point. For example in Ref. 10, a zero bias peak was observed. In this paper, we use the power, found from the heat balance equations, to calculate responsivity $S_{V}$ and NEP. In addition, we estimate the incoming power, radiated by the black body (BB) in one mode. The latter quantity limits the possible absorbed power from the top.

The sample is shown in Fig. 1. Four arrays, consisting of three bolometers each, are integrated into the cross slot antenna. The bolometers are connected in series for dc current and in parallel for high-frequency RF-current. The bolometer responses of opposite slots are summed up for dc read out. The details of the biasing circuitry are shown in the inset in Fig. 1. The presented design of cross-slot antenna is based on the original work for $550 \mathrm{GHz} .{ }^{11}$ For our purpose, 


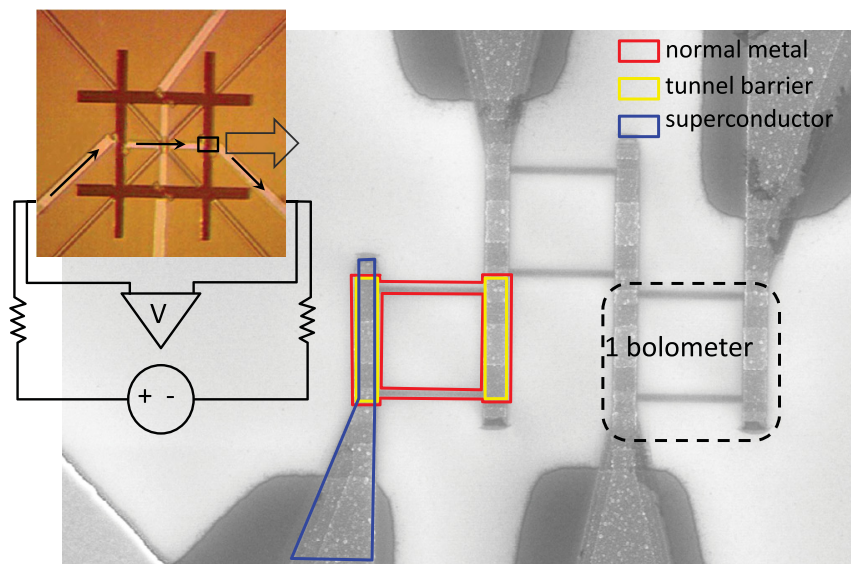

FIG. 1. Main: Three bolometers in one slot of the antenna (SEM image). Inset: cross-slot antenna in the optical microscope. The array of bolometers is biased through bias resistors.

the antenna has been scaled down to $350 \mathrm{GHz}$. The antenna is made of gold. The cold electron bolometer consists of a normal metal absorber $(\mathrm{N})$ made of aluminium with suppressed superconductivity, an aluminium oxide tunnel barrier(I), and aluminium electrodes (S) of the $40 \mathrm{~nm}$ superconducting thin film (SINIS junctions). All three layers are shown by colour frames in Fig. 1.

The main parts of the experimental setup (Fig. 2) are: the antenna with bolometers coupled to a silicon lens with a diameter of $4 \mathrm{~mm}$, the optical can with filters from one side, and the source of black body radiation attached to another cryostat plate. The sample in the can has been cooled down to $200 \mathrm{mK}$ and illuminated by a black body (BB) with temperature varying from $2.7 \mathrm{~K}$ to $47 \mathrm{~K}$.

Black body radiation was filtered by a set of filters fabricated at Cardiff University with a bandwidth of $33 \mathrm{GHz}$ and a central frequency of $350 \mathrm{GHz}$. Their transmission is $10^{-8}$

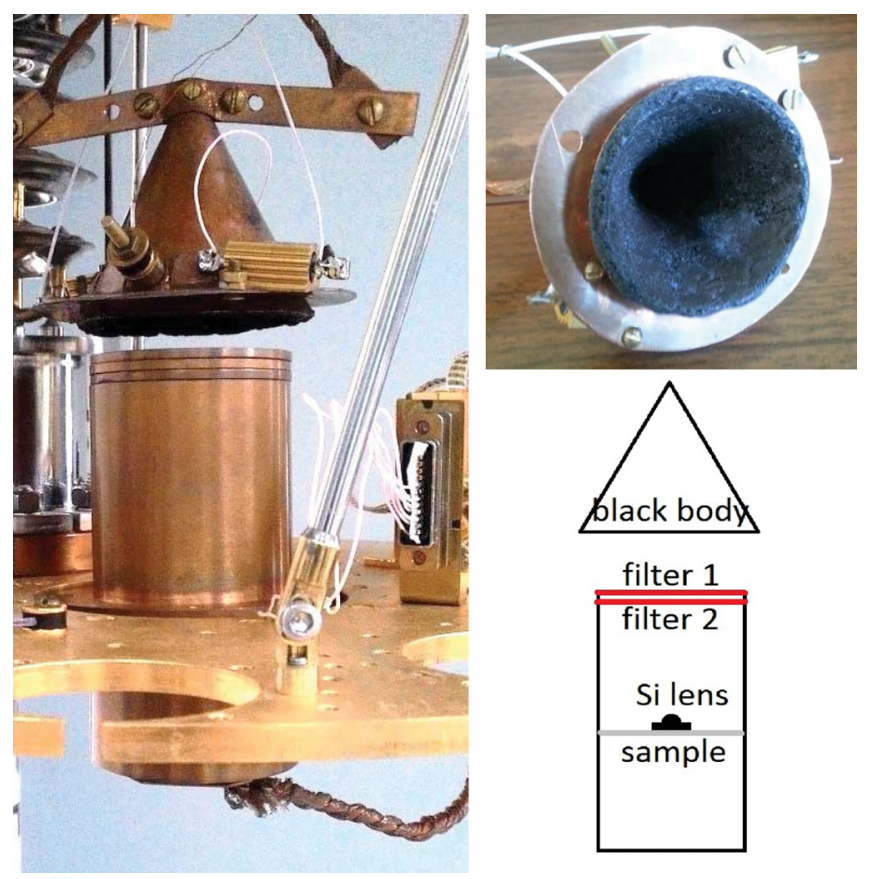

FIG. 2. The view of experimental setup, its schematic representation, and the black body surface. from 500 to $850 \mathrm{GHz}$ and $10^{-6}$ from 850 to $1200 \mathrm{GHz}$. The transmission above $1200 \mathrm{GHz}$ was not measured. The equivalent filters were mounted on the optical window of the balloon cryostat of BOOMERanG. ${ }^{12}$

In Fig. 3, the current-voltage characteristics of CEBs are shown for different $\mathrm{BB}$ temperatures. The figure also includes the electron temperature $T_{E}$ for each IV-curve, extracted from the tunneling current across the NIS junction

$$
I=\frac{1}{e R_{N}} \int_{-\infty}^{\infty} v(\varepsilon)\left[\frac{1}{\exp \left(\frac{\varepsilon-e V}{\tau_{E}}\right)+1}-\frac{1}{\exp \left(\frac{\varepsilon}{\tau_{S}}\right)+1}\right] d \varepsilon
$$

Here, $v(\varepsilon)$ is a density of states in the superconductor with Dynes parameter $\gamma$ :

$$
v(\varepsilon)=\left|\operatorname{Re}\left[\frac{\varepsilon / \Delta-i \gamma}{\sqrt{(\varepsilon / \Delta-i \gamma)^{2}-1}}\right]\right| .
$$

Four parameters are required to find $\tau_{E}$ (normalized $T_{E}$ ) from Eq. (1): the critical temperature $T_{c}$ of the superconductor, the normal state resistance of the junction $R_{N}$, the normalized temperature of electrons in superconductor $\tau_{S}$, and the parameter $\gamma$. Eq. (1) has a vague dependence on $\tau_{S}$, which is equal to phonon temperature, and $\gamma$, so that only two parameters, $T_{c}$ and $R_{N}$, have to be measured in order to get accurate values of $T_{E}$ from IV-curves. For this sample, we have used the following values: $T_{c}=1.47 \mathrm{~K}, R_{N}=8 \mathrm{k} \Omega$ (for the array), and $\gamma=5 \times 10^{-5}$.

Two heat balance equations, for the normal absorber ${ }^{13}$ and for superconductor electrodes, respectively, can be written in the following form: ${ }^{14-16}$

$$
\left\{\begin{array}{l}
P_{N}+P_{L O A D}+2 \beta P_{S}=P_{E-P H}+2 P_{C O O L}, \\
\epsilon(1-\beta) P_{S}=P_{E-P H, S} .
\end{array}\right.
$$

Here, $P_{N}$ is the Joule heating of the normal absorber, $P_{C O O L}$ is the cooling power of the NIS junction, $P_{L O A D}$ is the absorbed power of radiation (the source of photon noise),

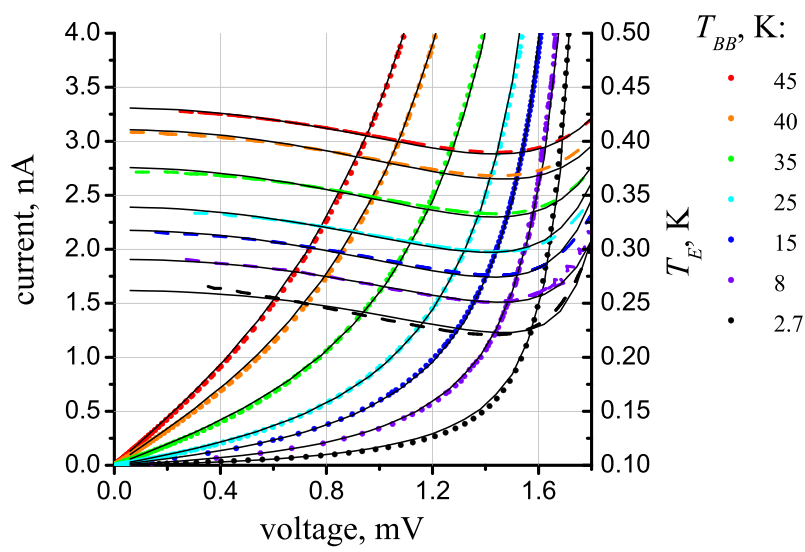

FIG. 3. Measured current (dots), the electron temperature, calculated from Eq. (1) (dashed curves), and the result of fitting from Eq. (3) (black solid curves) as functions of bolometer voltage for different black body temperatures $T_{B B}$. 
and $P_{S}$ is a power brought to the superconductor by hot electrons from the absorber. Its fraction $2 \beta$ returns to the normal metal, another fraction $\epsilon(1-\beta)$ heats the electron subsystem in the superconductor near the barrier, and the rest is transferred away and dissipates in the substrate. $P_{E-P H}=\Sigma V\left(T_{E}^{5}-T_{P H}^{5}\right)$ is the electron phonon coupling in the normal metal. $P_{E-P H, S}=0.98 \Sigma_{S} V_{S}\left(T_{S}^{5}-T_{P H}^{5}\right) e^{-\Delta\left(T_{S}\right) / \tau_{S}}$ is the electron-phonon coupling in the superconductor. ${ }^{17}$

Some parameters are known from direct measurements or technological process: the absorber resistance $R_{a b s}$ $=110 \Omega$, the absorber volume $V=0.02 \mu \mathrm{m}^{-3}$, and the volume of the superconducting electrode $V_{S}=2.5 \mu \mathrm{m}^{-3}$. Whereas the material constants $\Sigma_{N}=1.25 \mathrm{nW} \mu \mathrm{m}^{-3} \mathrm{~K}^{-5}$, $\Sigma_{S}=0.3 \mathrm{nW} \mu \mathrm{m}^{-3} \mathrm{~K}^{-5}$, the backtunneling coefficient $\beta=0.3$, degree of heating of superconducting electrons $\epsilon$, and the absorbed power $P_{L O A D}$ are found from the fit. The material constants are in agreement with literature data ${ }^{18}$ for both superconducting and normal aluminium.

The easiest for fitting and the most trustworthy from the point of view of the absorbed power is a region approximately from 0.1 to 0.6 of the voltage gap of the whole structure (combined gap for 3 bolometers in series). There, the absorbed power exceeds the other heating powers and therefore can be found with high accuracy. Closer to the gap, the fitting is more tricky since the other heating terms start dominating. Nevertheless, the results of our fitting with two heat balance equations are in good agreement with the experiment for the whole range of voltages shown in Fig. 3. For example, the electron temperature, obtained from the fit, coincides with values, found directly from IV-curves using expression (1), with the accuracy better than $3 \mathrm{mK}$.

In Fig. 4, the spectra of experimental voltage noise of CEBs biased at $2 \mathrm{nA}$ are presented. One can see that the change in the $\mathrm{BB}$ temperature increases the average noise level. The peaks at frequencies multiple of $50 \mathrm{~Hz}$ do not interfere the observation of the spectral flat region above $60-70 \mathrm{~Hz}$.

In Fig. 5, the experimental noise at a frequency of $120 \mathrm{~Hz}$ versus electron temperature is presented at a fixed bias current of $2 \mathrm{nA}$. The electron temperature of the absorber has been increased in two ways: (1) by heating of the sample holder and (2) by heating of the black body. The

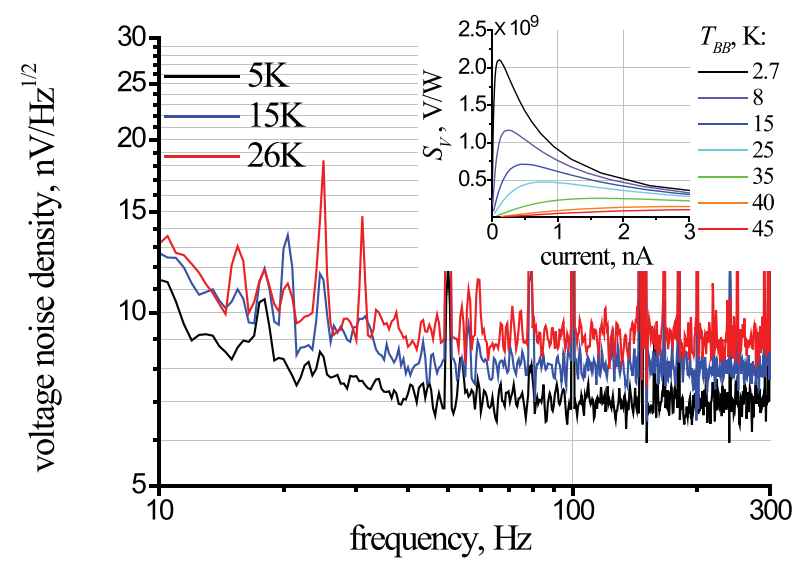

FIG. 4. Voltage noise at bias current $2 \mathrm{nA}$ for various black body temperatures, $T_{P H}=200 \mathrm{mK}$. Inset: The responsivity versus bias current for various black body temperatures.

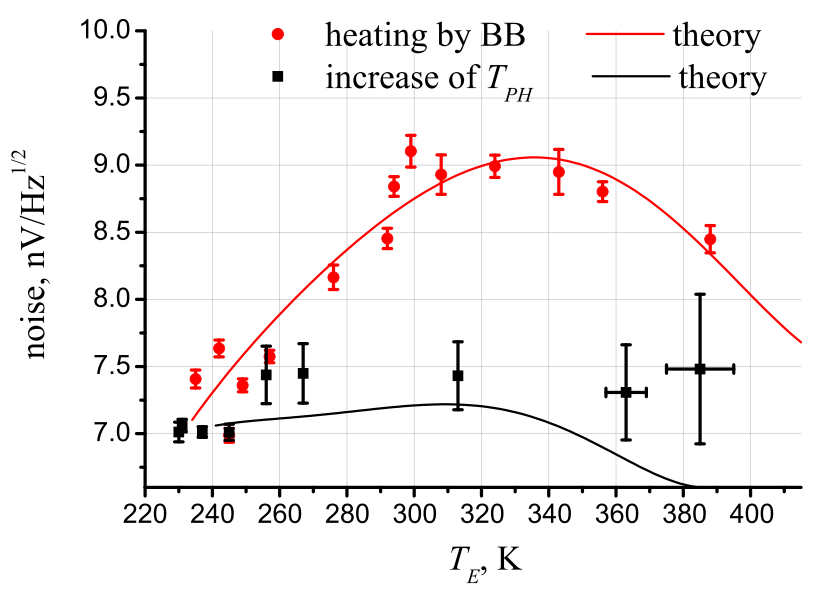

FIG. 5. Noise versus electron temperature for black body heating (red circles) at $T_{P H}=200 \mathrm{mK}$ and plate heating (black squares). Solid curves: fitting results. Bias current is $2 \mathrm{nA}$.

former heats the phonons and then the electrons via a weak link between the two systems; the latter should heat the electrons much more efficiently since the power is absorbed directly by the electrons. The different character of noise behaviour in these two cases is a signature of the presence of photon noise in the latter experiment. When $T_{E}$ in two experiments coincides, the differential resistance and the amplifier noise are the same as well, but the total noise is different. The reason, why the theory is outside the errors of the measurements in the first experiment (black data), is a lowfrequency noise due to a temperature-induced drift at large temperatures of the cryostat plate. The higher the temperature set point of the coldest plate with the sample, the harder the stabilization of the cryostat. The voltage noise of bolometers is superimposed on a slow voltage drift, which for a significant measurement time exceeds the noise of bolometers. Despite we measure noise at a frequency of $120 \mathrm{~Hz}$ to avoid the flicker noise, this frequency is still influenced by the drift.

The noise in cold-electron bolometers has several components: noise of the electron-phonon interaction, SIN junction noise (includes shot noise of current through a tunnel junction, noise of heat flow, and correlation between them), amplifier noise, and photon noise. All components are written below in the listed order in terms of NEP:

$$
\begin{aligned}
N E P_{\text {amp }}^{2} & =\frac{\delta V^{2}+(R \delta I)^{2}}{S_{V}^{2}}, \\
N E P_{E-P H}^{2} & =10 k_{B} \Sigma V\left(T_{E}^{6}+T_{P H}^{6}\right), \\
N E P_{\text {sin }}^{2} & =\partial P^{2}+\frac{\partial I^{2}}{\left(\partial I / \partial V \cdot S_{V}\right)^{2}}-2 \frac{\partial P \partial I}{\partial I / \partial V \cdot S_{V}}, \\
N E P_{\text {photon }}^{2} & =2 h \nu P_{L O A D}+\frac{P_{L O A D}^{2}}{\Delta \nu} .
\end{aligned}
$$

Here, $\delta V=4.7 \mathrm{nV} / \sqrt{\mathrm{Hz}}$ is the voltage noise and $\delta I=12 \mathrm{fA} /$ $\sqrt{\mathrm{Hz}}$ is the current noise at $120 \mathrm{~Hz}$ of the amplifiers AD745. The amplifier noise was calibrated using a resistor and agrees with the passport data at $120 \mathrm{~Hz}: 5 \mathrm{nV} / \sqrt{\mathrm{Hz}}$ and $9 \mathrm{fA} / \sqrt{\mathrm{Hz}}$.

The noise dependence on the bolometer voltage is presented in Fig. 6. The noise was measured at a frequency of $120 \mathrm{~Hz}$ and $T_{B B}=20 \mathrm{~K}$, which corresponds to $0.72 \mathrm{pW}$ of 


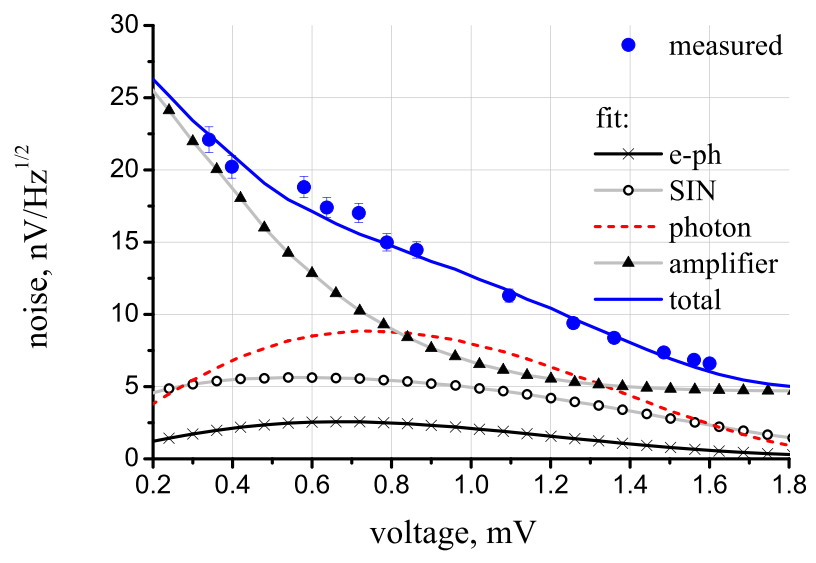

FIG. 6. Measured voltage noise at $120 \mathrm{~Hz}$ and various components of theoretical noise versus bolometer voltage $V$ for BB temperature $20 \mathrm{~K}, T_{P H}=200 \mathrm{mK}$.

absorbed power. At this power level, the photon noise is significant and higher than the amplifier noise in the range of 0.85-1.32 $\mathrm{mV}$. In order to maximize the photon noise component in comparison with other components, the operation point has to be slightly higher than the half gap of the whole structure.

In Fig. 7, the main result of the paper is shown: the experimental noise versus absorbed power for a fixed bias point $2 \mathrm{nA}$ decomposed in terms (4) with the help of theoretical analysis. The theory and the experiment are in good agreement. The photon noise component exceeds all other components separately at power loads ranging from 0.5 to 2 $\mathrm{pW}$. The least noise component, which can even be neglected, is the electron-phonon noise. The sum contribution of two other terms (SIN and amplifier) is slightly larger than the photon noise. Nevertheless, one can clearly see that the shape of the total noise originates from the photonic component.

Let us consider the component of the photon noise $\delta V_{\text {photon }}=N E P_{\text {photon }} \cdot S_{V}$. Photon NEP has root dependence on power $P_{L O A D}$ for $\nu \gg \Delta \nu$, see Eq. (4). This leads to a sharp increase in the photon noise from minimum absorbed power to $P_{L O A D}=1 \mathrm{pW}$, Fig. 7. Then, the dependence $S_{V}\left(P_{L O A D}\right)$ starts to play a decisive role $\left(S_{V}\right.$ for different power loads is shown in the inset of Fig. 4). The experiment

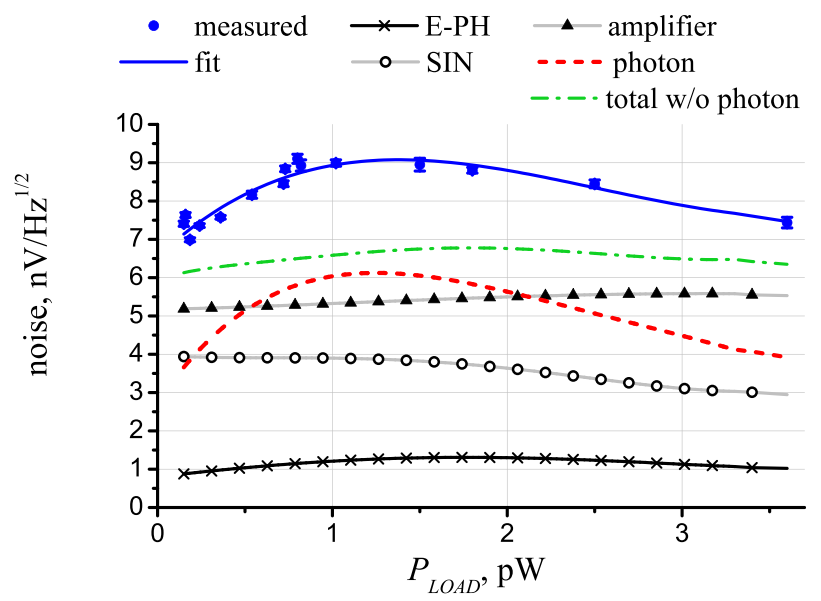

FIG. 7. Various components of noise versus absorbed power. Bias current $2 \mathrm{nA}$, and $T_{P H}=200 \mathrm{mK}$.

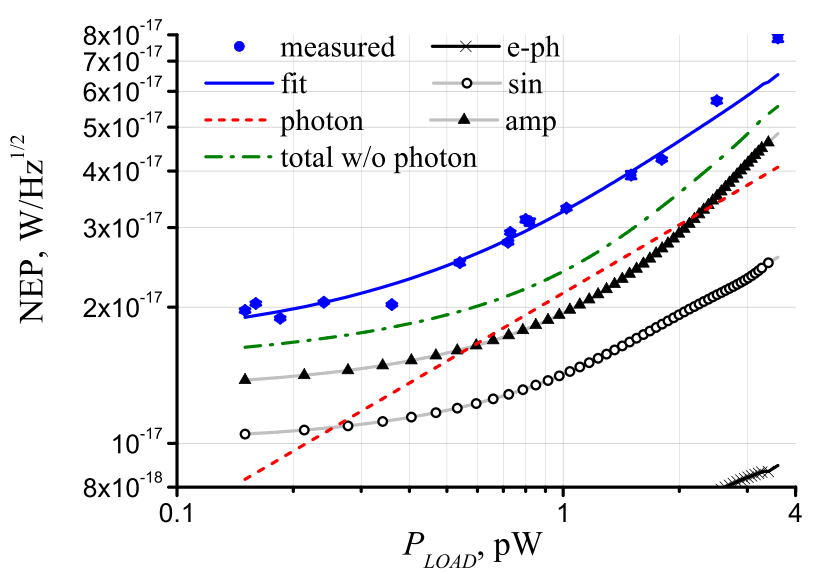

FIG. 8. Various components of NEP as a function of absorbed power. Bias current $2 \mathrm{nA}$ and $T_{P H}=200 \mathrm{mK}$.

shows that starting from $P_{L O A D}=1 \mathrm{pW}$ the responsivity decreases by a factor of three, resulting in a decline of the photon noise, Fig. 7. Thus, the experimental dependence of the noise in Fig. 7, confirmed by the theory, indicates that the bolometers are extremely sensitive to the photon noise at the background of internal noise of the system.

The noise equivalent power versus power load for the considered bolometer array is shown in Fig. 8. As can be seen, this array performs closely to the photon limited regime at power loads ranging from 0.5 to $2 \mathrm{pW}$. This is one of the main features of cold-electron bolometers: ${ }^{3,5}$ the size of the bolometer array (the number of parallel-series connections) is designed to achieve the optimum performance in the appropriate range of the external power load. In particular, the main criterion is the low system noise in comparison with the photon noise. A further decrease in the total noise can be made by a decrease in the amplifier noise component. It can be made by a change in read-out electronics to the acscheme or to the correlation scheme as in Refs. 7 and 8.

We have found that the efficiency of absorption for the whole structure in our case is around $30 \% .^{9}$ In order to get the incident power, $P_{L O A D}$ should be divided accordingly to the efficiency of absorption $P_{\text {incident }}=P_{L O A D} / 0.3$. In our experiment, we do not encounter the problem of excess power discussed in Ref. 19. However, we have found that in order to fit IV-curves, the parameters $\beta$ and $\epsilon$, responsible for returning power and for superconducting electrode heating, have to be changed gradually with the increase in $T_{b b}$. We assume that this is due to nonequilibrium effects in superconducting leads, described, for example, in Ref. 20.

We have shown both experimentally and theoretically that the cold electron bolometers are sensitive to the photon noise. The responsivity of bolometers $S_{V}=d V / d P_{L O A D}$ at the bias point decreases from $5 \times 10^{8}$ to $1.5 \times 10^{8} \mathrm{~V} / \mathrm{W}$ with the absorbed power. NEP increases from $2 \times 10^{-17} \mathrm{WHz}^{-1 / 2}$ to $6 \times 10^{-17} \mathrm{WHz}^{-1 / 2}$ correspondingly. The least ratio between internal CEB NEP (without photon noise) and $\mathrm{NEP}_{P H}$ observed in our experiments is below 1.1, while between total NEP and $\mathrm{NEP}_{P H}$, it is 1.5 for the absorbed power of $1-2 \mathrm{pW}$.

The authors would like to thank Paolo de Bernardis, Silvia Masi, and Mikhail Tarasov for stimulating 
discussions, Sumedh Mahashabde, Ernst Otto and Alexander Abashin for help in the sample fabrication, and Evgeniy Skorokhodov for the SEM image of the sample using facilities of the Common Research Center of IPM RAS. The work was supported by Swedish National Space Board, SNSB, and Russian Science Foundation (Project No. 16-1910468). Samples were fabricated in the Nanotechnology Center of Chalmers University of Technology and measured in the Center of Cryogenic Nanoelectronics of NNSTU.

${ }^{1}$ Planck and BICEP2/Keck Collaborations, Phys. Rev. Lett. 114(10), 101301 (2015).

${ }^{2}$ L. Kuzmin, SNED Proc. Vol. 1, Naples, Italy, May 28-June 1, 2001, pp. 145-154 (2002).

${ }^{3}$ L. S. Kuzmin, in Bolometers: Cold-Electron Bolometer, edited by A. G. U. Perera (InTech, 2012), p. 77. ISBN 978-953-51-0235-9.

${ }^{4}$ M. Salatino, P. de Bernardis, L. S. Kuzmin, S. Mahashabde, and S. Masi, J. Low Temp. Phys. 176, 323 (2014).

${ }^{5}$ L. S. Kuzmin, J. Phys.: Conf. Ser. 97, 012310 (2008).

${ }^{6}$ M. A. Tarasov, L. S. Kuzmin, V. S. Edelman, S. Mahashabde, and P. de Bernardis, IEEE Trans. Appl. Supercond. 21, 3635 (2011).

${ }^{7}$ T. L. R. Brien, P. A. R. Ade, P. S. Barry, C. Dunscombe, D. R. Leadley, D. V. Morozov, M. Myronov, E. H. C. Parker, M. J. Prest, M. Prunnila et al., Appl. Phys. Lett. 105, 043509 (2014).
${ }^{8}$ T. L. R. Brien, P. A. R. Ade, P. S. Barry, C. J. Dunscombe, D. R. Leadley, D. V. Morozov, M. Myronov, E. H. C. Parker, M. J. Prest, M. Prunnila et al., J. Low Temp. Phys. 184, 231 (2016).

${ }^{9}$ A. S. Mukhin, A. V. Gordeeva, L. S. Revin, A. E. Abashin, A. A. Shishov, A. L. Pankratov, S. Makhashabde, and L. S. Kuzmin, Radiophys. Quantum Electron. 59, 754 (2016).

${ }^{10}$ L. Kuzmin, I. Agulo, M. Fominsky, A. Savin, and M. Tarasov, Supercond. Sci. Technol. 17, S400 (2004).

${ }^{11}$ G. Chattopadhyay and J. Zmuidzinas, IEEE Trans. Antennas Propag. 46, 736 (1998).

${ }^{12}$ S. Masi, P. Ade, J. Bock, J. Bond, J. Borrill, A. Boscaleri, P. Cabella, C. Contaldi, B. Crill, P. de Bernardis et al., Astron. Astrophys. 458, 687 (2006).

${ }^{13}$ D. Golubev and L. Kuzmin, J. Appl. Phys. 89, 6464 (2001).

${ }^{14}$ S. Mahashabde, A. Sobolev, A. Bengtsson, D. Andrn, M. A. Tarasov, M. Salatino, P. de Bernardis, S. Masi, and L. S. Kuzmin, IEEE Trans. Terahertz Sci. Technol. 5, 145 (2015).

${ }^{15}$ G. S. O'Neil, Ph.D. thesis, University of Colorado, 2011.

${ }^{16}$ M. Saleh, M.S. thesis, Chalmers University of Technology, 2014.

${ }^{17}$ A. V. Timofeev, C. Pascual Garcia, N. B. Kopnin, A. M. Savin, M. Meschke, F. Giazotto, and J. P. Pekola, Phys. Rev. Lett. 102, 017003 (2009).

${ }^{18}$ F. Giazotto, T. T. Heikkila, A. Luukanen, A. M. Savin, and J. P. Pekola, Rev. Mod. Phys. 78, 217 (2006).

${ }^{19}$ J. Bueno, P. J. de Visser, S. Doyle, and J. J. A. Baselmans, J. Low Temp. Phys 176, 1089 (2014).

${ }^{20}$ A. S. Vasenko and F. W. J. Hekking, J. Low Temp. Phys. 154, 221 (2009). 